\title{
Composite Learning for Trajectory Tracking Control of Robot Manipulators with Output Constraints
}

\author{
Dianye Huang \\ Key Laboratory of Autonomous \\ Systems and Networked Control \\ College of Automation Science \\ and Engineering, \\ South China University of Technology \\ Guangzhou 510640, China.
}

\author{
Chenguang Yang \\ Key Laboratory of Autonomous \\ Systems and Networked Control \\ College of Automation Science \\ and Engineering, \\ South China University of Technology \\ Guangzhou 510640, China \\ Email: cyang@ieee.org
}

\author{
Yongping Pan \\ Department of Biomedical Engineering \\ National University of Singapore \\ Singapore 117583, Singapore
}

\author{
Shilu Dai \\ Key Laboratory of Autonomous Systems and Networked Control \\ College of Automation Science and Engineering, \\ South China University of Technology \\ Guangzhou 510640, China
}

\author{
Zhaojie Ju \\ School of Computing \\ University of Portsmouth \\ Portsmouth PO13HE UK
}

\begin{abstract}
In this paper, a trajectory tracking scheme for robot manipulators with unknown dynamics is investigated, with the consideration of output constraints as well as small bounded external disturbances. Firstly, a modified backstepping control scheme is employed to control the robot manipulators where in the first step of the design a tan-type barrier Lyapunov candidate is chosen in order to tackle the constraint problem. Secondly, the philosophy of dynamic surface control is incorporated to implement the calculation of prediction errors, which can also reduce "explosion of complexity" of the backstepping scheme. In addition, composite learning is introduced for a better estimation of unknown parameters, and for canceling out the uncertainties of the robot manipulators. Stability analysis shows that the proposed control scheme guarantees a small bounded tracking error with parameter convergence in the absence of the stringent persistent excitation condition. Finally, a simulation is conducted and the results demonstrate the superiority of the proposed controller in the aspects of tracking capability and parameter estimation.
\end{abstract}

Keywords-Barrier Lyapunov function (BLF); composite learning control (CLC); output constraints; dynamic surface control (DSC); robot manipulators

\section{INTRODUCTION}

Direct interaction between robots and environment has received increasing attention in recent years [1], [2], [3]. Even though in the application of interactive tasks, robot control still has to satisfy certain limits, including constraints on position, velocity, acceleration etc., violation of which may cause instability, degradation or even system damages [4]. Therefore, it is vital to design a robot manipulator controller which subjects to the constraints, at the same time, suppresses the effect of uncertainties for better tracking performance.
Dynamic Surface Control(DSC) is a well known control scheme for nonlinear system[5]. It improves the adaptive backstepping control( $\mathrm{ABC}$ ) which suffers from the problem of "explosion of complexity" by applying a first order filter to the virtual input control[6]. However, tackling with the constraint problem is out of the range of traditional DSC. The backstepping scheme combined with Barrier Lyapunov Function(BLF) is one of the existing methods to handle the effect of constraints, which can guarantee the constraint satisfactions [7]. The principle of this technique is to design a dedicate Lyapunov function that behaves infinite energy if the system states approaching sufficiently close to the predefined boundary. It is worth to note that the control law designed based on BLF generally imports inevitable lumped uncertainties when combining with Adaptive Backstepping Control(ABC). Approximation Technique based on neural network such as Fuzzy Neural Network(FNN) demonstrates powerful performances of handling uncertainties in nonlinear system control[8]. However, it only proofs to command the system states into a bounded compact set.

In the control design, it should be guaranteed that the tracking and estimating errors converges to an arbitrarily small region around 0 . To this end, an integrated scheme Composite Adaptive Control(CAC) has been developed in [9], where a parametric update law that consists of simultaneous tracking error and prediction errors was employed to identifying models for better tracking and estimation performance. Its superior performance such as faster parameter convergence and smaller tracking error, has been demonstrated by various applications[10], [11], [12] in the past decade. Meanwhile, 
the system guarantees parameter convergence if and only if the Persistence Excitation(PE) condition is met[13], which is too stringent to be satisfied. Recently, Concurrent Learning has been proposed to relax the PE condition to Interval Excitation (IE). However, in the design of Concurrent Learning, the singular value maximization requires an exhaustive searching of the data stack and derivation requirements of the system states. Consequently, Composite Learning is proposed for further relaxation in the aspect implementation[14], [15]. It utilizes the recorded historical data and concurrent data to generate the prediction error as well as the tracking error, which will be used to update the estimated parameters[15]. This method has been successfully applied to many applications, such as the control of the inverted pendulums, the multi-constraint nonlinear systems and the quadrotors [16], [11], [17]. Considering the advantages of CAC and practical implementation of Composite Learning Control(CLC), it is promising and feasible to integrate the composite learning into the controller.

In this paper, a trajectory tracking controller for robot manipulators is developed with the consideration of output constraints as well as small bounded external disturbances. The BLF is utilized to tackle with the constraint problem combing with dynamic surface control scheme. And the Composite learning is introduced to cancel out the uncertainties. The contribution of this paper is the development of a control scheme that successfully merges the constraints boundaries into the dynamic surface control and introduces the composite learning method to estimate the parameters of unknown dynamics from the historical prediction error and simultaneous tracking error.

The structure of this paper is given as follows: Section 2 gives some preliminaries. The constrained robot manipulator controller is designed in Section 3. Simulations will be carried out in Section 4. Finally, Section 5 concludes this paper. Some notations will be used in this paper, where $\lambda_{\min (\max )}(\cdot)$ denotes the minimal(maximal) eigenvalue of $\cdot, \operatorname{sgn}(\cdot)$ stands for the sign function, $\operatorname{diag}(\cdot)$ represents the diagonal matrix with $\cdot$ be its entries, $\|\cdot\|$ denotes the Euclidean norm of $\cdot \odot$ is the dot-product operator, where $a \in \mathbb{R}^{n}, b \in \mathbb{R}^{n}, c=a \odot b, c_{i}=a_{i} b_{i}, i=1,2, \ldots, n$.

\section{PRELIMINARIES}

\section{A. System descriptions}

Considering a robot manipulator with $n$ degree of freedom(DOF), the dynamics in joint space can be established as follow:

$$
M(q) \ddot{q}+C(q, \dot{q}) \dot{q}+G(q)+\tau_{d i s t}=\tau+\tau_{e}
$$

where $q, M(q), C(q, \dot{q}), G(q)$ denote the angle configuration, the inertia, Coriolis and centrifugal matrices, the vector of torque due to the effect of the gravity, respectively. $\tau$ represents the applied control torque. $\tau_{\text {ext }}$ and $\tau_{\text {dist }}$ stand for the torques that are applied to the plant due to the interaction forces, unknown disturbances, respectively. Properties of the manipulators are exploited in the previous literature [18]:
Property I. $\dot{M}(q)-2 C(q, \dot{q})=S$ is skew-symmetric, $\xi^{T} S \xi=0, \forall \xi \in \mathbb{R}^{n}$.

Property II. $M(q), C(q, \dot{q})$ and $G(q)$ are first differentiable, $\forall q, \dot{q} \in \mathbb{R}^{n}$

Property III. There exists a constant vector $P$ consisting of unknown parameters. It depends on the described robot manipulator and subjects to $M(q) a+C(q, \dot{q}) v+G(q)=$ $H(q, \dot{q}, a, v) P$, where $H(q, \dot{q}, a, v)$ is a known regression matrix.

The output constraints are formulated as below:

$$
\left|q_{i}-q_{r i}\right|<k_{i}, \forall t>0,(i=1,2, \ldots, n)
$$

where $k_{i}$ is a positive constant, $q_{r i}$ represents the reference trajectory of the $i$-th joint. The output of the robot manipulator should remain within the set $\varrho:=\left\{q \in \mathbb{R}^{n}|| q_{i}-q_{r i} \mid<\right.$ $\left.k_{i}, \forall t>0\right\} \subset \mathbb{R}^{n}, i=1,2, \ldots, n$. A tan-type Barrier Lyapunov Function(BLF) is chosen as [8]:

$$
V(\zeta)=\frac{k^{2}}{\pi} \tan \left(\frac{\pi \zeta^{2}}{2 k^{2}}\right),|\zeta(0)|<k
$$

where $\zeta \in \mathbb{R}$ is a scalar variable and $k$ is user-defined positive constant constraint. The projection operator is introduced for the purpose of circumventing parameter drift in the absence of PE [19].

$$
\rho(w, \varpi)= \begin{cases}\varpi, & \|w\|<\omega_{a} \text { or } \\ \varpi-\frac{w w^{T}}{\|w\|^{2}} \varpi, & \|w\|=\omega_{a} \& w^{T} \varpi \leq 0 \\ \text { otherwise. }\end{cases}
$$

where $\omega_{a}$ is a user-defined parameter based on priori knowledge.

\section{B. Assumptions and lemmas}

Assumption 1: The reference trajectory $q_{r}$ and its first time derivative $\dot{q}_{r}$ are continuous and bounded.

Assumption 2: No unmeasured interaction forces are applied to the plant. Under this assumption, without lose of generality let $\tau_{e}=0$ in (1). In variable structure system, the disturbances, such as viscous and Coulomb friction forces $f_{v} \dot{q}+f_{c} \operatorname{sgn}(\dot{q})$ are generally consider to be the function of system states[20]. Therefore, it is reasonable to assume that the disturbances are bounded and the following inequality holds:

$$
\left\|\tau_{\text {dist }}\right\| \leq d_{1}\left\|\xi_{2}\right\|+d_{2}\left\|\xi_{1}\right\|+\left\|d_{3}\right\|
$$

where $d_{1,2} \in \mathbb{R}^{+}$are some constants and $\xi_{1,2}$ are states indicating the tracking performance which will be defined later. We also assume that the disturbances varies slowly $\dot{\tau}_{\text {dist }} \approx 0$ and $d_{3} \in \mathbb{R}^{n}$ is an uncertain variable that can be estimated as time evolutes.

Lemma 1 [21]: Lyapunov candidate function $\mathrm{V}(\mathrm{t})$ is positive, continuously differentiable with bounded initial state $\mathrm{V}(0)$. If the following equation is satisfied:

$$
\dot{V} \leq-\rho V+a
$$

where $\rho>0, a>0$, the solution of signals of which $V(t)$ consisting are uniformly bounded. 
Lemma 2 [22] : For filter (13) with assumption 1,2, given any $\mu \in \mathbb{R}^{+}$, there exists an sufficiently small $\lambda$ in (13) such that within finite time $T_{\alpha},\left|\beta_{2 i}-\alpha_{2 i}\right| \leq \mu, \forall t \in\left[0, T_{\alpha}\right]$, $i=1,2, \ldots, n$.

Lemma 3 [8]: Define $\theta$ as a constant column vector to be estimated and $\hat{\theta}$ as its estimated counterpart. $\tilde{\theta}:=\theta-\hat{\theta}$, $\Gamma=\operatorname{diag}\left(\gamma_{1}, \gamma_{2}, \ldots, \gamma_{n}\right), \gamma_{i}>0$ for $i=1, \ldots, n$. Then the following inequality holds:

$$
\tilde{\theta}^{T} \Gamma \hat{\theta} \leq-\frac{1}{2} \tilde{\theta}^{T} \Gamma \tilde{\theta}+\frac{1}{2} \theta^{T} \Gamma \theta
$$

Proof: $\tilde{\theta}^{T} \Gamma \hat{\theta}=\sum_{i=1}^{n} \gamma_{i} \tilde{\theta}_{i}\left(\theta_{i}-\tilde{\theta}_{i}\right)$. Using Young's inequality, there is $\tilde{\theta}_{i} \theta_{i} \leq \frac{1}{2} \tilde{\theta}_{i} \tilde{\theta}_{i}+\frac{1}{2} \theta_{i} \theta_{i}$. Thus, we have $\tilde{\theta}^{T} \Gamma \hat{\theta} \leq$ $\sum_{i=1}^{n} \gamma_{i}\left(-\frac{1}{2} \tilde{\theta}_{i} \tilde{\theta}_{i}+\frac{1}{2} \theta_{i} \theta_{i}\right)=-\frac{1}{2} \tilde{\theta}^{T} \Gamma \tilde{\theta}+\frac{1}{2} \theta^{T} \Gamma \theta$.

Lemma 4 [14]: Let $z$ be the signal whose first time derivative $\dot{z}$ is practically unavailable. A second order filter can be employed to estimate it:

$$
\begin{aligned}
& \dot{\xi}_{1}=\xi_{2} \\
& \dot{\xi}_{2}=-2 \zeta \omega \hat{\xi}_{2}+\omega^{2}\left(z-\xi_{1}\right)
\end{aligned}
$$

with initial condition, $\xi_{1}(0)=\hat{z}(0), \xi_{2}(0)=\hat{\dot{z}}(0)$, where $\xi_{1}, \xi_{2}$ are estimated counterparts of $z, \dot{z}$.

\section{CONTROL DESIGN}

Consider a manipulator with $n$ DOF under the condition of output constraints $\left|\xi_{1 i}\right|<k_{c i},(i=1,2, \ldots, n)$. The robotic dynamics in (1) is rewritten under Assumption 1 as:

$$
\begin{aligned}
& \dot{x}_{1}=x_{2} \\
& \dot{x}_{2}=M^{-1}(q)\left[\tau-\tau_{\text {dist }}-C(q, \dot{q}) \dot{q}-G(q)\right]
\end{aligned}
$$

where $x_{1}:=q, x_{2}:=\dot{q}$. Define the error signals as $\xi_{1}:=$ $x_{1}-\alpha_{1}, \xi_{2}:=x_{2}-\alpha_{2}$, where $\alpha_{1,2}$ are the virtual control inputs and $\alpha_{1} \equiv q_{r}$. For saving space, $M, C, G$ are the abbreviation of $M(q), C(q, \dot{q}), G(q)$, respectively. It won't be hard to yield the error equation as follow:

$$
M \dot{\xi}_{2}+C \xi_{2}=\tau-\tau_{d i s t}-M \dot{\alpha}_{2}-C \alpha_{2}-G
$$

\section{A. Model based controller}

In this section, we assume that the model of the plant is exactly known. Considering constraints in (2) $\left|\xi_{1 i}\right|<$ $k_{c i}, i=1,2, \ldots, n$, we choose the tan-type Barrier Lyapunov Function candidate as [8]:

$$
V_{1}(t)=\sum_{i=1}^{n} \frac{k_{c i}^{2}}{\pi} \tan \left(\frac{\pi \xi_{1 i}^{2}}{2 k_{c i}^{2}}\right)+\frac{1}{2} \xi_{2}^{T} M \xi_{2}
$$

taking its time derivative along Property I, II, Assumption 1, (10) and the definition of error signals yields:

$$
\begin{aligned}
\dot{V}_{1}(t)= & \sum_{i=1}^{n} \frac{\xi_{1 i}\left(\xi_{2 i}+\left(\beta_{2 i}+\tilde{\alpha}_{2 i}\right)-\dot{\alpha}_{1 i}\right)}{\cos ^{2}\left(\frac{\pi \xi_{1 i}^{2}}{2 k_{c i}^{2}}\right)}+ \\
& \xi_{2}^{T}\left(\tau-\tau_{\text {dist }}-\left(M \dot{\alpha}_{2}+C \alpha_{2}+G\right)\right)
\end{aligned}
$$

where $\tilde{\alpha}_{2 i}:=\beta_{2 i}-\alpha_{2 i}$.

A modified Dynamic Surface Control(DSC) scheme is adopted, where BLF as (3) is chosen as the Lyapunov candidate in the first step of the design. In the second step, in order to reduce the 'explosion of complexity', a first-order filter is employed with initial condition $\alpha_{2}(0)=\beta_{2}(0)$ :

$$
\lambda \dot{\alpha}_{2}+\alpha_{2}=\beta_{2}
$$

where $\beta_{2}$ is the virtual control input which will be designed later and $\alpha_{2}$ is its filtered counterpart. Considering Lemma 1 , we can construct (12) according to the framework of (6) through appropriately designing $\beta_{2}$ and $\tau_{1}$ as below:

$$
\beta_{2}=\tau_{\text {aux }}-\mu \cdot \operatorname{sgn}\left(\xi_{1}\right)+\dot{\alpha}_{1}
$$

where $\mu \geq \sup \left(\left|\tilde{\alpha}_{2 i}\right|\right), i=1,2, \ldots, n$,

$$
\tau_{\text {aux }}=-K\left[\begin{array}{c}
\frac{k_{c 1}^{2}}{\xi_{11} \pi} \sin \left(\frac{\pi \xi_{11}^{2}}{2 k_{c 1}^{2}}\right) \cos \left(\frac{\pi \xi_{11}^{2}}{2 k_{c 1}^{2}}\right) \\
\cdots \\
\frac{k_{c n}^{2}}{\xi_{1 n} \pi} \sin \left(\frac{\pi \xi_{1 n}^{2}}{2 k_{c n}^{2}}\right) \cos \left(\frac{\pi \xi_{1 n}^{2}}{2 k_{c n}^{2}}\right)
\end{array}\right]
$$

The control input $\tau$ is designed as:

$$
\tau_{1}=\tau_{c}+\tau_{d i s t}-K_{p} \xi_{2}+M \dot{\alpha}_{2}+C \alpha_{2}+G
$$

where $\tau_{c}=-\left[\begin{array}{c}\frac{\xi_{11}}{\cos ^{2}\left(\frac{\pi \xi_{11}^{2}}{2 k_{c 1}^{2}}\right)} \\ \frac{\ldots}{\operatorname{\xi }_{1 n}} \\ \cos ^{2}\left(\frac{\pi \xi_{1 n}^{2}}{2 k_{c n}^{2}}\right)\end{array}\right], K=\operatorname{diag}\left(k_{1}, k_{2}, \ldots, k_{n}\right)$, $K_{p}=\operatorname{diag}\left[k_{p 1}, k_{p 2}, \ldots, k_{p n}\right]$ as positive constant diagonal matrices. Substituting (18) and (16) into (12) and combining with Lemma 2, we have:

$$
\dot{V}_{1}<-\sum_{i=1}^{n} k_{i} \frac{k_{c i}^{2}}{\pi} \tan \left(\frac{\pi \xi_{1 i}^{2}}{2 k_{c i}^{2}}\right)-\xi_{2}^{T} K_{p} \xi_{2}<-\rho_{1} V_{1}
$$

where $\rho_{1}$ is positive and subjects to $\rho_{1}=$ $\min \left(\lambda_{\min }(K), 2 \lambda_{\min }\left(K_{p}\right) / \lambda_{\max }(M)\right)$. Therefore, $\dot{V}_{1}(t)$ is negative-definite, which ensures the stability of the closed loop system.

Observing (14), $\mu \cdot \operatorname{sgn}\left(\xi_{1}\right)$ is designed via the Lyapunov stability analysis. Although the DSC scheme relaxes the condition that $\beta_{2}$ should be differentiable, as the parameters of the first order filter choosing smaller, the negative effect of sign function becomes nonnegligible. Remind that in sliding mode control the chattering problem can be eliminated by the saturation function where $\operatorname{sgn}(s)=s$, if $|s|<b$ is satisfied. Consequently, the control law is smoothed at the same time guarantees the stability of the system. Considering that the constrained controller guarantees the tracking error $\xi_{1}$ stay within small predefined boundaries. Therefore, we may replace $\mu \cdot \operatorname{sgn}\left(\xi_{1}\right)$ with $\mu \xi_{1}$ but saturation function to reduce the undesired effect of sign function:

$$
\beta_{2}=\tau_{\text {aux }}-\mu \cdot \xi_{1}+\dot{\alpha}_{1}
$$

\section{$B$. Controller with respect to uncertainties}

Practically, the controller (16) cannot be implemented because of the lumped uncertainty $M(q) \dot{\alpha}_{2}+C(q, \dot{q}) \alpha_{2}+G$. Thus, we employ composite learning to deal with it. According to Property III, there is:

$$
H\left(q, \dot{q}, \alpha_{2}, \dot{\alpha}_{2}\right) P=M(q) \dot{\alpha}_{2}+C(q, \dot{q}) \alpha_{2}+G
$$


where $\mathrm{P}$ are the plant parameters to be estimated. Composite learning is adopted for parameter convergence. The update law is given by [15]:

$$
\dot{\hat{P}}=\rho\left(\hat{P},-\Gamma\left(H^{T} \xi_{2}+\kappa \epsilon\right)\right)
$$

where $\kappa \in \mathbb{R}^{+}$is the weighting constant balancing the contribution of the simultaneous tracking error with the prediction error and $\Gamma \in \mathbb{R}^{n \times n}$ is the learning rate. For more detailed calculation process of the modelling error, please refer to [15] in Section III A. Remark 2. We denote:

$$
H_{\alpha}(t):=H\left(\dot{q}(t), q(t), \dot{\alpha}_{2}(t), \alpha_{2}(t)\right)
$$

and $\Phi(t)$ is given by:

$$
\Phi(t):=\int_{t-\tau_{d}}^{t} H_{\alpha}^{T}(t) H_{\alpha}(t) d \tau
$$

Where $\tau_{d}$ stands for the integrate time. Define prediction error as $\Phi(T) \tilde{P}$. $\epsilon(\mathrm{t})$ is denoted as modelling error the calculation of which is illustrated as following:

Generally, the prediction error cannot be obtained exactly. It is of great necessity to find a way calculating it. Firstly, we take a further examine on the prediction error.

$$
\Phi\left(t^{*}\right) \tilde{P}(t)=\frac{1}{\tau_{d}} \int_{t^{*}-\tau_{d}}^{t^{*}} H_{\alpha}^{T}(\tau) \cdot\left(H_{\alpha}(\tau) P\right) d \tau-\Phi(t) \hat{P}(t)
$$

The equation (10) can be expressed as follow:

$[M C G] \cdot\left[\dot{\xi}_{2}, \xi_{2}, 0\right]^{T}=\tau-\tau_{d i s t}+[M C G] \cdot\left[\dot{\alpha}_{2}, \alpha_{2}, 1\right]^{T}$

Since $\dot{\xi}_{2}$ is unavailable, a unit second-order linear filter is employed as shown in Lemma 4 to estimate it. In addition, $\tau_{\text {dist }}$ is also unavailable, therefore, an update law for $\tau_{\text {dist }}$ estimation is designed during the stability analysis. One can divide (23) into a computable term $\Phi(t) P_{c}(t)$ and mismatching uncertainties $\varepsilon_{d}(t)$, that is, $\Phi(t) \tilde{P}(t)=\Phi(t) \tilde{P}_{c}(t)-\varepsilon_{d}(t)$.

$$
\begin{aligned}
\Phi\left(t^{*}\right) P_{c}(t)= & \frac{1}{\tau_{d}} \int_{t^{*}-\tau_{d}}^{t^{*}} H_{\alpha}^{T}(\iota) \cdot\left(\tau-\hat{\tau}_{d i s t}\right) \hat{\Lambda} d \iota- \\
& \Phi\left(t^{*}\right) \hat{P}(t) \\
\varepsilon_{d}\left(t^{*}\right)= & \frac{1}{\tau_{d}} \int_{t^{*}-\tau_{d}}^{t^{*}} H_{\alpha}^{T}(\iota) \tilde{\tau}_{d i s t} \Lambda d \iota+\Delta_{\Lambda}(\omega)
\end{aligned}
$$

where $\hat{\Lambda}=\left(\left[\dot{\xi}_{2}+\dot{\alpha}_{2}, \xi_{2}+\alpha_{2}, 1\right]^{T}\right)^{\dagger} \cdot\left[\dot{\alpha}_{2}, \alpha, 1\right]^{T}, \hat{\Lambda}=\left(\left[\hat{\dot{\xi}}_{2}+\right.\right.$ $\left.\left.\dot{\alpha}_{2}, \hat{\xi}_{2}+\alpha_{2}, 1\right]^{T}\right)^{\dagger} \cdot\left[\dot{\alpha}_{2}, \alpha, 1\right]^{T}, \tilde{\tau}_{\text {dist }}:=\tau_{\text {dist }}-\hat{\tau}_{\text {dist }} . \Delta_{\Lambda}(\omega)$ is the sum of the rest mismatching uncertainties. Note that, $\left\|H_{\alpha}(t)\right\|<\psi,\left\|\tilde{\tau}_{\text {dist }}\right\|<\varphi,\|\Lambda\|<v,\left\|\Delta_{\Lambda}(\omega)\right\|<\varsigma$, there is $\left\|\varepsilon_{d}(t)\right\|<\psi \tau_{d} \varphi v+\varsigma<\bar{\varepsilon}_{d}$. If $\omega$ is chosen sufficiently small, as time evolutes $\xi_{2} \rightarrow 0, \Delta_{\Lambda} \approx 0 . \Phi(t) P_{c}(t)$ is used to form the update law of composite learning.

$$
\epsilon\left(t^{*}\right)= \begin{cases}\Phi\left(t^{*}\right) \tilde{P}_{c}(t), & \text { if } t \geq\left(\tau_{d}+T_{a}\right) \& \Phi(t) \geq \sigma I \\ \Phi\left(T_{e}\right) \tilde{P}_{c}(t), & \text { otherwise. }\end{cases}
$$

where

$$
T_{e}= \begin{cases}t, & \text { if } t \geq\left(\tau+T_{a}\right) \& \Phi(t) \geq \sigma I \\ T_{e}, & \text { otherwise. }\end{cases}
$$

where we define that $\Phi\left(T_{e}\right)=0$ if $t \leq \tau+T_{a}, T_{a}$ is the least time that filters' errors reduced to an acceptable range. The control input is redesigned as:

$$
\tau=\tau_{c}-\bar{d}_{2} \xi_{3}-K_{p} \xi_{2}+H\left(q, \dot{q}, \dot{\alpha}_{2}, \alpha_{2}\right) \hat{P}+\hat{d}_{3}
$$

Where $\xi_{3}:=\left|\xi_{1}\right| \odot \operatorname{sgn}\left(\xi_{2}\right), \quad \bar{d}_{2}>d_{2}, \lambda_{\min }\left(K_{p}\right)>d_{1}$. Choose the Lyapunov Function as:

$$
V(t)=V_{1}(t)+\frac{1}{2} \tilde{P}^{T} \Gamma^{-1} \tilde{P}+\frac{1}{2} \gamma^{-1} \tilde{d}_{3}^{T} \tilde{d}_{3}
$$

Taking the time derivative of it along (1) and (28) with update law (20) and

$$
\dot{\hat{d}}_{3}=-\gamma\left(\xi_{2}+\eta \hat{d}_{3}\right)
$$

yields:

$$
\begin{aligned}
\dot{V}(t) \leq & -\sum_{i=1}^{n} k_{i} \frac{k_{c i}^{2}}{\pi} \tan \left(\frac{\pi \xi_{1 i}^{2}}{2 k_{c i}^{2}}\right)-\xi_{2}^{T} K_{p} \xi_{2}-\frac{1}{2} \eta \tilde{d}_{3}^{T} \tilde{d}_{3} \\
& -\kappa \tilde{P}^{T} \epsilon+\frac{1}{2} \eta d_{3}^{T} d_{3}
\end{aligned}
$$

Firstly, consider $t \in\left[0, T_{e}\right], \epsilon=0$, according to Lemma 1 , the closed-loop system is stable and the its relating signal $\chi$ converges to a bounded set. Secondly, for $t \in[T e, \infty]$, there exists $\Phi(t) \geq \sigma I$ such that $\epsilon(t)=\Phi\left(T_{e}\right) \tilde{P}_{c}(t)=$ $\Phi\left(T_{e}\right) \tilde{P}(t)+\varepsilon_{d}(t)$. Note that $\hat{P}, P \in \Omega_{c_{w}}$, one gets $\|\tilde{P}\| \leq 2 c_{w}$. There is:

$$
\begin{aligned}
\dot{V}(t) \leq & -\sum_{i=1}^{n} k_{i} \frac{k_{c i}^{2}}{\pi} \tan \left(\frac{\pi \xi_{1 i}^{2}}{2 k_{c i}^{2}}\right)-\xi_{2}^{T} K_{p} \xi_{2}-\frac{1}{2} \eta \tilde{d}_{3}^{T} \tilde{d}_{3} \\
& -\kappa \sigma \tilde{P}^{T} \tilde{P}(t)+\kappa \tilde{P}^{T} \varepsilon_{d}(t)+\frac{1}{2} \eta d_{3}^{T} d_{3} \\
\leq & -\kappa_{e} V(t)+\vartheta\left(\kappa_{e}, \omega, \kappa, \eta\right)
\end{aligned}
$$

where $\kappa_{e}=\min \left(\lambda_{\min }(K), \frac{2 \lambda_{\min }\left(K_{p}\right)}{\lambda_{\max }(M)}, \frac{2 \kappa \sigma}{\lambda_{\max }\left(\Gamma^{-1}\right)}, \eta \gamma\right)$, $\vartheta\left(\kappa_{e}, \omega, \kappa, \eta\right)=\left(2 \kappa c_{w} \bar{\varepsilon}_{d}+\frac{1}{2} \eta d_{3}^{T} d_{3}\right) / \kappa_{e}, \vartheta \in \mathbb{R}^{+}$. Therefore, we can conclude that consider a dynamic system (1) with constraints (2), applying control input in (28), update law in (20) and (30), the system relating signals $\chi\left(\xi_{1}, \xi_{2}, \tilde{P}, \tilde{d}_{3}\right)$ exponentially converge to a bounded set $\Omega_{c}=\left\{\chi \mid\|\chi\| \leq 2 V(0)+2 \vartheta / \kappa_{e}\right\}$. Note that through appropriately choosing the parameters $\kappa_{e}, \omega, \kappa, \eta, \vartheta$ can be made sufficiently small, such that $\chi$ will exponentially converge to a small region near 0 .

\section{Simulation}

A two-link planar manipulator as [20] shown in Fig. 1 is constructed to verify the validity of the proposed control scheme. Its dynamics is given by :

$$
\begin{aligned}
& {\left[\begin{array}{ll}
M_{11} & M_{12} \\
M_{12} & M_{22}
\end{array}\right]\left[\begin{array}{l}
\ddot{q}_{1} \\
\ddot{q}_{2}
\end{array}\right]+\left[\begin{array}{cc}
-c \dot{q}_{2} & -c\left(\dot{q}_{1}+\dot{q}_{2}\right) \\
c \dot{q}_{1} & 0
\end{array}\right] \times} \\
& {\left[\begin{array}{c}
\dot{q}_{1} \\
\dot{q}_{2}
\end{array}\right]+\left[\begin{array}{l}
\varrho_{1} g \\
\varrho_{2} g
\end{array}\right]=\left[\begin{array}{l}
\tau_{1} \\
\tau_{2}
\end{array}\right]}
\end{aligned}
$$



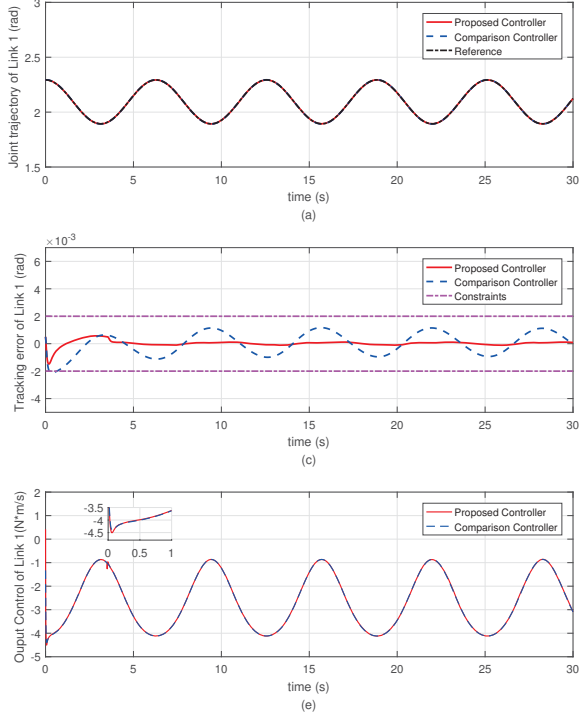
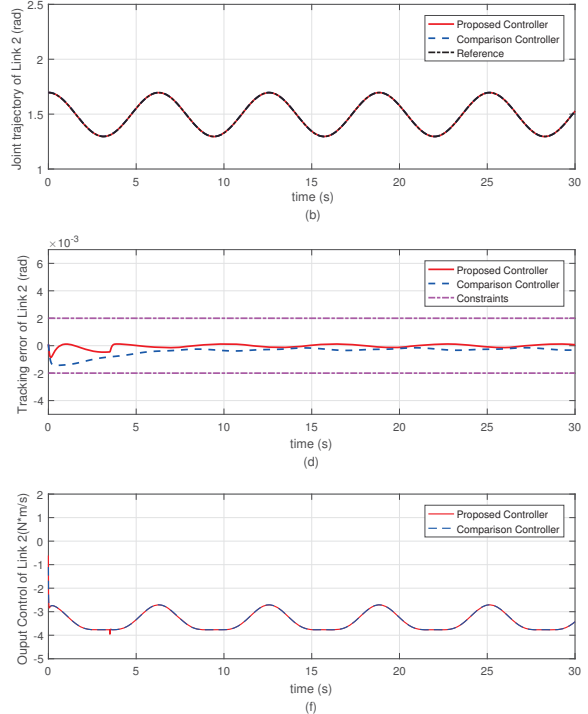

Fig. 2: The tracking performance of the "Proposed Controller" and "Comparison Controller"
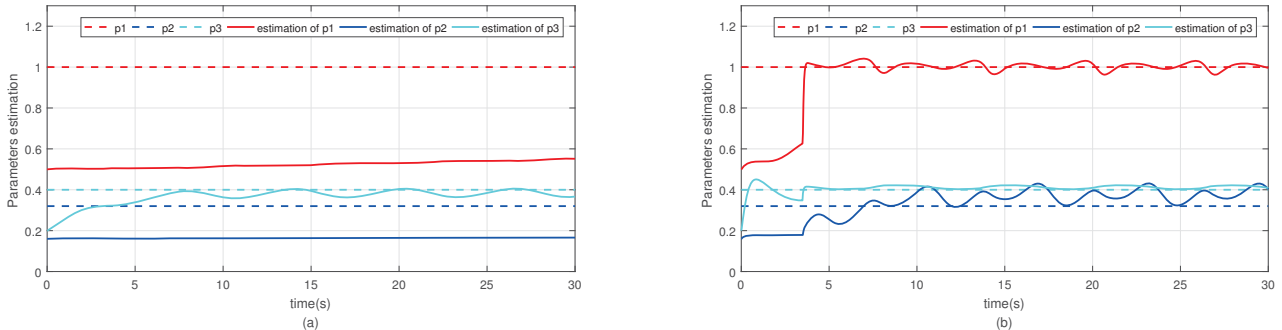

Fig. 3: The parameters estimation of the Proposed Controller and Comparison Controller

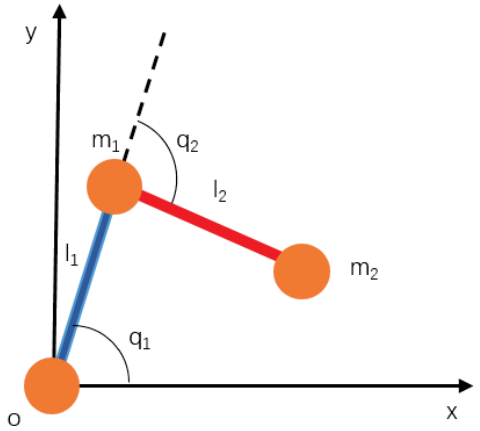

Fig. 1: A two-link manipulator model

where

$$
\begin{aligned}
M_{11} & =\left(m_{1}+m_{2}\right) l_{1}^{2}+m_{2} l_{2}^{2}+2 m_{2} l_{1} l_{2} \cos \left(q_{2}\right) \\
M_{12} & =m_{2} l_{2}^{2}+m_{2} l_{1} l_{2} \cos \left(q_{2}\right) \\
M_{22} & =m_{2} l_{2}^{2} \\
c & =m_{2} l_{1} l_{2} \sin \left(q_{2}\right) \\
\varrho_{1} & =\left(m_{1}+m_{2}\right) l_{1} \cos \left(q_{2}\right)+m_{2} l_{2} \cos \left(q_{1}+q_{2}\right) \\
\varrho_{2} & =m_{2} l_{2} \cos \left(q_{1}+q_{2}\right)
\end{aligned}
$$

where $l_{1}=1 \mathrm{~m}, l_{2}=0.8 \mathrm{~m}, m_{1}=0.5 \mathrm{~kg}, m_{2}=0.5 \mathrm{~kg}$, $g=9.8, r=g / l_{1}$. The parameters to be estimated are $P=\left[\left(m_{1}+m_{2}\right) l_{1}^{2}, m_{2} l_{2}^{2}, m_{2} l_{1} l_{2}\right]^{T}=[1.0,0.32,0.4]^{T}$. The reference trajectory is set to be $q_{r}(t)=[1.9935,1.3961]^{T}+$ $0.3 \cos (t)$. The initial configuration of the manipulator is $q_{0}=[2.293,0.1696]^{T} \mathrm{rad} . \tau_{\text {dist }}=[0.1+0.1 \sin (t), 0.1+$ $0.1 \sin (t)]^{T}$ will be applied to the manipulator as disturbances.

A conventional backstepping controller is introduced for comparison, which is referred to "Comparison Controller". Its control law and corresponding update law are given as follows:

$$
\begin{aligned}
\tau & =H\left(q, \dot{q}, \alpha_{2}, \dot{\alpha}_{2}\right) \hat{P}-K_{p} z_{2}-z_{1}-\bar{\mu} \operatorname{sat}\left(z_{2}\right) \\
\dot{\hat{P}} & =-\Gamma_{c}\left(H^{T} z_{2}+W_{c} \hat{P}\right), V=\frac{1}{2} z_{1}^{T} z_{1}+\frac{1}{2} z_{2}^{T} M z_{2} \\
z_{1} & :=x_{1}-x_{d}, z_{2}:=x_{2}-\alpha_{2}, \alpha_{2}=\dot{x}_{d}-\lambda_{s} z_{1}
\end{aligned}
$$

The constraints in (2) is selected as $k_{1}=0.002, k_{2}=$ 0.002. Parameters of the "Comparison Controller" are well tuned, and $\hat{P}(0)=[0.5,0.16,0.2]^{T}$. Estimation parameters of the "Proposed Controller" are set to be $\hat{P}(0)=$ $[0.5,0.16,0.2]^{T}$ for fair comparison. It is crucial to note that the initial value of estimated parameters in (20) cannot be 
set to be zeros, since the control scheme is a combination of constraint controller and composite learning method. At the beginning of tracking task, the error would swiftly achieve to the predefined boundaries if the uncertainties cannot be partly cancelled, which may result in an infinity output control that the actuators can not be physically satisfied, thus we initialize the parameters $50 \%$ of true values.

The simulation is carried out in MATLAB 2016a running on Windows 10, setting ode5 solver with fixed step size $0.001 \mathrm{~s}$. The simulation results shown in Fig. 2,3 illustrate the performance of the "Comparison Controller" and the "Proposed Controller" in the aspects of tracking error and parameter estimation in the presence of disturbances.

In Fig. 2 (c)(d), the proposed controller tracks the reference trajectory better and always stays within the predefined boundaries. We can see from Fig. 2 (e) that constraints violation is avoided due to the swift response of the output torque when the tracking error getting close to the boundaries. Obviously, in Fig. 3, the "Proposed Controller" estimates the unknow parameters better than the "Comparison Controller". Noted that the reference trajectory does not fully excite the system, therefore the estimation performance of the "Comparison Controller" is far from satisfaction. However, even under these circumstances, the "Proposed Controller" can still boundely converges around the true value, which shows its superiority. Therefore, on the whole, we can conclude that the "Proposed Controller" outperforms the "Comparison Controller".

\section{Conclusion}

In this paper, we develop a novel trajectory tracking controller for robot manipulators with the considerations of output constraints as well as small external disturbances. Firstly, the backstepping control scheme is employed as a fundamental scheme to be modified. Secondly, to tackle with the constraints problem, BLF is introduced as lyapunov candidate in the first step. Thirdly, to release the "explosion of complexity" from which the backstepping control suffered, the philosophy of the dynamic surface control is referred to. Eventually and most importantly, composite learning is adopted to handle with the uncertainties. Stability analysis shows that the proposed controller guarantees exponentiallike convergence of the trajectory tracking and parameter estimation errors. And the simulation demonstrates its validity.

\section{ACKNOWLEDGMENT}

This work was supported in part by the National Nature Science Foundation under Grant 61473120, in part by the Science and Technology Planning Project of Guangzhou under Grant 201607010006, in part by the State Key Laboratory of Robotics and System under Grant SKLRS-2017-KF-13, and in part by the Fundamental Research Funds for the Central Universities under Grant 2017ZD057.

\section{REFERENCES}

[1] C. Yang, K. Huang, H. Cheng, Y. Li, and C. Y. Su, "Haptic identification by elm-controlled uncertain manipulator," IEEE Transactions on Systems, Man, and Cybernetics: Systems, vol. 47, no. 8, 2017.
[2] C. Yang, Y. Jiang, Z. Li, W. He, and C. Y. Su, "Neural control of bimanual robots with guaranteed global stability and motion precision," IEEE Transactions on Industrial Informatics, vol. 13, no. 3, 2016.

[3] C. Yang, X. Wang, Z. Li, Y. Li, and C.-Y. Su, "Teleoperation control based on combination of wave variable and neural networks," IEEE Transactions on Systems, Man, and Cybernetics: Systems, vol. 47, no. 8, 2016.

[4] W. He, A. O. David, Z. Yin, and C. Sun, "Neural network control of a robotic manipulator with input deadzone and output constraint," IEEE Transactions on Systems, Man, and Cybernetics: Systems, vol. 46, no. 6, pp. 759-770, 2016.

[5] P. P. Yip and J. K. Hedrick, "Adaptive dynamic surface control: A simplified algorithm for adaptive backstepping control of nonlinear systems," International Journal of Control, vol. 71, no. 5, pp. 959979, 1998.

[6] Y. Pan and H. Yu, Dynamic Surface Control via Singular Perturbation Analysis. Pergamon Press, Inc., 2015.

[7] Y.-J. Liu and S. Tong, "Barrier lyapunov functions for nussbaum gain adaptive control of full state constrained nonlinear systems," Automatica, vol. 76, pp. 143-152, 2017.

[8] W. He and Y. Dong, "Adaptive fuzzy neural network control for a constrained robot using impedance learning," IEEE Transactions on Neural Networks and Learning Systems, vol. 29, no. 4, 2017.

[9] W. Li, "Composite adaptive control of robot manipulators," in Leee/rsj International Conference on Intelligent Robots and Systems, 1989, pp. 293-298.

[10] P. M. Patre, S. Bhasin, Z. D. Wilcox, and W. E. Dixon, "Composite adaptation for neural network-based controllers," IEEE Transactions on Automatic Control, vol. 55, no. 4, pp. 944-950, 2010.

[11] T. Sun, Y. Pan, C. Yang, T. Sun, Y. Pan, and C. Yang, "Composite adaptive locally weighted learning control for multi-constraint nonlinear systems," Applied Soft Computing, 2017.

[12] Y. Pan, T. Sun, and H. Yu, "Composite adaptive dynamic surface control using online recorded data," International Journal of Robust and Nonlinear Control, vol. 26, no. 18, pp. 3921-3936, 2016.

[13] J. Sun and J. Sun, Robust Adaptive Control. Prentice-Hall, Inc., 1995.

[14] Y. Pan, M. J. Er, L. Pan, and H. Yu, "Composite learning from model reference adaptive fuzzy control," in International Conference on Fuzzy Theory and ITS Applications, 2015, pp. 91-96.

[15] Y. Pan and H. Yu, "Composite learning from adaptive dynamic surface control," IEEE Transactions on Automatic Control, vol. 61, no. 9, pp. 2603-2609, 2016.

[16] Y. Pan, L. Pan, and H. Yu, "Composite learning control with application to inverted pendulums," pp. 232-236, 2015.

[17] B. Xu, "Composite learning finite-time control with application to quadrotors," IEEE Transactions on Systems Man and Cybernetics Systems, vol. PP, no. 99, pp. 1-10, 2017.

[18] R. Ortega, J. A. L. Perez, P. J. Nicklasson, and H. Sira-Ramirez, Passivity-Based Control of Euler-Lagrange Systems: Mechanical, Electrical and Electromechanical Applications. Springer Science \& Business Media, 2013.

[19] S. J. Cusumano, "Adaptive robust control," Circuits and Systems .proceedings.proceedings of Midwest Symposium on, vol. 2, no. 2, pp. $645-648,1988$.

[20] C.-Y. Su and T.-P. Leung, "A sliding mode controller with bound estimation for robot manipulators," IEEE Transactions on Robotics and Automation, vol. 9, no. 2, pp. 208-214, 1993.

[21] S. S. Ge and C. Wang, "Adaptive neural control of uncertain mimo nonlinear systems," IEEE Transactions on Neural Networks, vol. 15, no. 3, pp. 674-692, 2004.

[22] W. Dong, J. A. Farrell, M. M. Polycarpou, and M. Sharma, "Command filtered adaptive backstepping," in American Control Conference, 2010, pp. 105-110. 